\title{
Efecto de la Adición de Goma Gelana sobre las Propiedades de Barrera y Físico Mecánicas de Biopelículas Binarias
}

\author{
Rafael E. González-Cuello*, Jaime Pérez-Mendoza y Luis A. García-Zapateiro \\ Facultad de Ingeniería. Departamento de Operaciones Unitarias. Grupo de Investigación Ingeniería de \\ Fluidos Complejos y Reología de Alimentos (IFCRA). Universidad de Cartagena, Av. Del Consulado, Calle \\ 30 № 48-157., Cartagena de Indias - Colombia (e-mail: rgonzalezc1@unicartagena.edu.co)
}

${ }^{*}$ Autor a quien debe ser dirigida la correspondencia.

Recibido Ago. 24, 2016; Aceptado Oct. 18, 2016; Versión final Nov. 21, 2016, Publicado Feb. 2017

\begin{abstract}
Resumen
El objetivo del presente estudio fue caracterizar las propiedades físico mecánicas y de barrera al vapor de agua de biopelículas (BP) a base de carboximetilcelulosa (CMC) y goma xantana (GX) formuladas con varias concentraciones de goma gelana de bajo acilo (GBA). Las BP fueron elaboradas por el método de casting y posteriormente fueron determinadas la permeabilidad al vapor de agua (PVA), elongación al quiebre (EQ), resistencia a la tensión (RT), humedad y transparencia. Los resultados obtenidos indicaron que las BP con menores concentraciones de GBA mostraron propiedades más deseables con respecto a las demás formulaciones, ya que se producen BP con baja humedad y PVA. Además presentan elevados valores de $E Q$ y son más transparentes. Estos resultados son realmente prometedores y sugieren la posibilidad de utilizar combinaciones terciarias de CMC, GX y GBA como una fuente de empaque biodegradable para aplicaciones alimentarias.
\end{abstract}

Palabras clave: biopelículas; elongación al quiebre, goma gelana; permeabilidad al vapor de agua; resistencia a la tensión

\section{Effect of Gellan Gum Addition on the Barrier and Physico mechanical Properties of Binary Biofilms} Abstract

The aim of this study was to characterize physico mechanical and water vapor barrier of biofilms based on carboxymethylcellulose (CMC) and xanthan gum (XG), formulated with various concentrations of low acyl gellan (LAG). The biofilms, were prepared by the casting method and then the water vapor permeability (WVP), elongation at break (EAB), tensile strength (TS), moisture and transparency were determined. The findings indicated that biofilms with low concentrations of LAG showed more desirable properties when is compared with the others formulations, because they yields biofilms with low moisture and WVP. In addition, these biofilms had high values of EAB and were more transparent. These results are promising and suggest the possibility of using tertiary mixtures of CMC, GX and LAG as a source of biodegradable packaging for food applications.

Keywords: biofilms; elongation at break; gellan gum; water vapor permeability; tensile strength 


\section{INTRODUCCIÓN}

El desarrollo de biopelículas biodegradables y comestibles para sustituir parcial o completamente los materiales poliméricos sintéticos se ha incrementado en las últimas décadas, debido a los problemas ambientales ocasionados por los materiales termoplásticos. Los materiales poliméricos utilizados como empaque de matrices alimentarias normalmente son polisacáridos, lípidos, proteínas y sus respectivas mezclas. Las biopelículas (BP) pueden actuar como una barrera a la migración de humedad, gases, aromas y solutos. Igualmente, pueden funcionar como acarreadores de ingredientes alimentarios (compuestos activos, agentes antimicrobianos, saborizantes y colorantes) extendiendo la vida útil y/o influenciando las propiedades mecánicas (resistencia y flexibilidad) de la matriz alimentaria (Kokoszka et al., 2010). En este sentido, las BP basadas en polisacáridos son vistas como potenciales sustitutos de los empaques sintéticos tradicionales y han sido caracterizadas por múltiples investigadores (Ahmadi et al., 2012; Ghasemlou et al., 2011), ya que son considerados barreras efectivas contra los gases y solutos por su densa matriz polimérica.

La carboximetilcelulosa $(\mathrm{CMC})$ es un polisacárido derivado de la celulosa con un residuo lineal aniónico de glucopiranosa $\beta$ (1-4) (Su et al., 2010). La CMC es originada por sustitución parcial de grupos hidroxilos por grupos carboxilos (Tong et al., 2008). Varios autores han reportado la importancia de producir BP a base de CMC debido a la posibilidad de obtener una matriz continua (Ghanbarzadeh y Almasi, 2011). La goma xantana (GX) es un heteropolisacárido que consiste de D-glucosa, D-manosa y ácido D-glucurónico. La GX es producida por Xantomonas campestris a través de fermentación aeróbica en cultivo sumergido (Guo et al., 2014) y sus dispersiones presentan un comportamiento reológico adecuado para la formación de BP (Baldwin et al., 2012). La goma gelana está formada por unidades de repetición de un tetrasacárido (1-3 $\beta$ D glucosa, 1,4 $\beta$ D ácido glucurónico, 1,4 $\beta$ D glucosa, $\alpha$-L ramnosa). La gelana nativa se conoce como gelana de alto acilo (GAA) porque presenta grupos acetato y glicerato en su residuo de glucosa. Cuando la GAA es expuesta a un fuerte tratamiento con álcali a elevadas temperaturas, los grupos acilo son hidrolizados y la gelana de bajo acilo (GBA) es obtenida. La GBA se caracteriza por formar geles firmes (González et al., 2012) y por su naturaleza aniónica puede formar geles en presencia de cationes.

Dentro de las desventajas que presentan las BP, se encuentran sus débiles propiedades mecánicas, las cuales normalmente son menores en comparación a los materiales sintéticos (Bourtoom, 2008). Por tanto, varias investigaciones han sido llevadas a cabo con el fin de mejorar la flexibilidad y resistencia (Hager et al., 2012). La adición de plastificantes como el glicerol, ha sido exitosamente utilizado en la producción de BP flexibles a base de polisacáridos (Ahmadi et al., 2012; Ghasemlou et al., 2011; Khazaei et al., 2014), también se ha evaluado la incorporación de agentes entrecruzantes. Sin embargo, estos pueden tener problemas de toxicidad (Cao et al., 2007) y por tal razón su aplicación no se ha extendido. Igualmente, se han estudiado las propiedades de empacado de películas celulósicas conteniendo pediocina (Pérez et al., 2013), así como las propiedades físico mecánicas y de barrera de goma gelana (Yang, et al., 2010). Sin embargo, no hay casi estudios concernientes al efecto de la GBA sobre las propiedades físico mecánicas y de barrera de BP a base de CMC y GX. Por consiguiente, el objetivo del presente estudio fue caracterizar las propiedades físico mecánicas y de barrera al vapor de agua de biopelículas binarias formuladas con varias concentraciones de goma gelana de bajo acilo.

\section{MATERIALES Y MÉTODOS}

Se explica la metodología de elaboración de las biopelículas y se detalla cómo se determina el espesor, el contenido de humedad y la transparencia de las biopelículas. Luego se explica cómo se determina la resistencia a la tensión y elongación al quiebre, y la permeabilidad del vapor de agua. Finalmente se explica cómo se realiza el análisis de los datos obtenidos.

\section{Elaboración de las biopelículas}

Las soluciones formadoras de las BP fueron preparadas de la siguiente manera: dispersiones de CMC (8\% $\mathrm{p} / \mathrm{v})$ conteniendo $\mathrm{GX}(5 \% \mathrm{p} / \mathrm{v})$, glicerol $(10 \% \mathrm{v} / \mathrm{v})$ y $30 \mathrm{mM}$ de Calcio fueron adicionadas con concentraciones crecientes de goma gelana de bajo acilo (GBA) $(0.1,0.3,0.5,0.7 \%$ p/v) bajo agitación constante a una temperatura de $90^{\circ} \mathrm{C}$ durante 10 minutos ajustando el $\mathrm{pH}$ a 4.0 con glucono $\delta$ lactona. Para la formación de las BP fueron vertidos volúmenes específicos de las soluciones anteriores en cajas de Petri. El secado se realizó en estufa a $30^{\circ} \mathrm{C}$ durante 45 horas aproximadamente asegurando uniformidad de las BP.

\section{Espesor, Contenido de humedad y transparencia de las biopelículas}

El espesor de las BP fue determinado utilizando un micrómetro con una exactitud de $0.01 \mathrm{~mm}$. Se tomaron cinco medidas de cada biopelículas: una en el centro y cuatro en sitios distanciados reportando los 
promedios aritméticos. La humedad fue estimada gravimétricamente en muestras de las BP de $2 \mathrm{~cm}$ mediante secado a $105^{\circ} \mathrm{C}$ en un horno durante $24 \mathrm{~h}$. La transparencia fue determinada a una longitud de onda de $560 \mathrm{~nm}$ utilizando un espectrofotómetro (Thomson Gold Spectrumlab 54). Las muestras de biopelículas fueron cortadas en rectángulos y colocadas directamente en celda del espectrofotómetro, el aire fue utilizado como referencia. El valor de transparencia fue calculado utilizando la siguiente ecuación:

$\operatorname{Tr}=A 560 / x$

Donde, A560 es la absorbancia a $560 \mathrm{~nm}$ y $\mathrm{x}$ es el espesor de las BP (mm). La mayor valor de transparencia representa la menor transparencia en las BP.

\section{Resistencia a la tensión y elongación al quiebre}

Después de obtener las BP se determinó la resistencia a la tensión (RT) y el porcentaje de elongación al quiebre (EQ) utilizando un texturometro Shimadzu modelo EZ-Test EZ-S empleando una geometría de mordazas a una velocidad de separación de $30 \mathrm{~mm} / \mathrm{min}$. Los valores de RT fueron calculados dividiendo la carga máxima $(\mathrm{N})$ por el área transversal $\left(\mathrm{m}^{2}\right)$ de las $\mathrm{BP}$ :

$R T(M P a)=\frac{P}{b x d}$

Donde $\mathrm{P}$ es la carga máxima $(\mathrm{N})$; b es el ancho de la muestra; y d es el espesor $(\mathrm{mm})$. El porcentaje de elongación fue determinado de la siguiente forma:

$E Q=\frac{l_{\max }}{l_{o}} \times 100$

Donde $I_{\max }$ es la elongación $(\mathrm{mm})$ al momento de la ruptura; y lo es longitud inicial $(\mathrm{mm})$. Todas las determinaciones fueron realizadas tres veces y se reportaron los promedios aritméticos

Permeabilidad del vapor de agua (PVA)

Esta propiedad fue determinada gravimétricamente conforme al método ASTM, 2010 con $75 \%$ de humedad relativa a $30^{\circ} \mathrm{C} \pm 1^{\circ} \mathrm{C}(13)$.

La PVA fue calculada utilizando la siguiente ecuación:

$P V A=\left[\frac{W}{t}\right] \cdot\left[\frac{\mathrm{x}}{\Delta \mathrm{P} \cdot \mathrm{A}}\right]$

Donde, $W$ es el cambio de peso debido a la absorción de agua ( $\mathrm{g}$ ); t es el tiempo en días; $\mathrm{x}$ es el espesor de la biopelículas $(\mathrm{mm}) ; \mathrm{A}$ corresponde al área de la $\mathrm{BP}\left(\mathrm{m}^{2}\right) ; \triangle \mathrm{P}$ es la diferencia de presiones parciales a través de la biopelículas. $\Delta \mathrm{P}$ fue calculada utilizando la siguiente ecuación:

$\Delta P=S *\left(R_{1}-R_{2}\right)$

Donde, $\mathrm{S}$ es la presión del vapor de agua saturada a $25^{\circ} \mathrm{C}(3166 \mathrm{kPa}) ; \mathrm{R} 1$ es la humedad relativa en el desecador $(0,82) ; \mathrm{R}^{2}$ es la humedad relativa en el interior $(0,18)$ expresada en fracciones (Pérez et al., 2013).

\section{Análisis de Datos}

Los resultados fueron sometidos a análisis normal de varianza (ANOVA un factor) utilizando la prueba tukey ( $5 \%$ de significancia) utilizando el programa de computo SPSS (Statistical Package for the Social Sciences) versión 17.0 para Windows (SPSS Inc. Chicago, IL, USA).

\section{RESULTADOS Y DISCUSIÓN}

Para una mejor organización, los resultados y su discusión se presentan en cinco subsecciones: (i) Espesor de las biopelículas; (ii) Contenido de humedad; (iii) Transparencia de las biopelículas; (iv) Permeabilidad al vapor de agua); y (v) Resistencia a la tensión y elongación al quiebre. 


\section{Espesor de las biopelículas}

El espesor de las BP estuvo entre 0.16 y $0.18 \mathrm{~mm}$ observándose diferencias significativas $(P<0.05)$ entre las BP sin GBA (control) y conteniendo GBA en varias concentraciones (0.1, $0.3,0.5$ y $0.7 \% \mathrm{p} / \mathrm{v}$ ). Espesores menores $(0.054$ a $0.060 \mathrm{~mm})$ fueron reportados por Dick et al., (2015) en BP elaboradas a partir del mucilago chia con diferentes proporciones de glicerol. Las diferencias significativas apreciadas generalmente son ocasionadas por los distintos contenidos de sólidos de cada solución. Las BP conteniendo una mayor concentración de GBA $(0.7 \mathrm{p} / \mathrm{v})$ presentaron los mayores valores de espesor $(0.18$ $\mathrm{mm}$ ), estos resultados sugieren que la GBA junto a la CMC y GX origina una red polimérica compacta, debido a un entrecruzamiento dentro de la matriz, resultando así un leve incremento en los valores de espesor. Igualmente, es importante mencionar que las mencionadas diferencias significativas pueden ser ocasionadas por el efecto que tiene la goma gelana sobre la reología de las dispersiones, principalmente la viscosidad (González et al., 2012). Nur Hazirah et al., (2016) observaron variaciones en el espesor de BP a base de gelatina y CMC cuando incrementaron la concentración de GX. Por otro lado, Mostafavi et al., (2016) no encontraron diferencias significativas en el espesor de biopelículas a base de goma tragacanto y goma algarrobo, atribuyendo tal comportamiento al estricto control durante el proceso de secado de las muestras. Es importante mencionar que en el presente estudio, el volumen para cada solución formadora de BP fue controlado durante el proceso de elaboración para asegurar valores similares de espesor. En tal sentido, las BP obtenidas fueron lo suficientemente delgadas para ser calificadas como películas según ASTM 1985 (American Society for Testing and Materials (ASTM) quienes establecieron espesores menores a $0.25 \mathrm{~mm}$, así como por Embuscado y Huber (2009) quienes mencionan que para ser consideradas películas deben poseer espesores menores a $0.3 \mathrm{~mm}$. Es de gran relevancia controlar el espesor de las BP debido a que normalmente este parámetro puede afectar tanto las propiedades físico mecánicas como de barrera de las BP elaboradas. En tanto, al disminuir las variaciones significativas en el espesor, se garantiza que los cambios apreciados en las propiedades evaluadas serán atribuidos principalmente a las variaciones efectuadas en la formulación de las BP.

\section{Contenido de humedad}

Los valores de contenido de humedad en las BP a base de mezclas de CMC/GX presentaron variaciones conforme aumentaban las concentraciones de GBA (tabla 1). Los mayores valores de humedad fueron encontrados en BP elaboradas a base de $8.0 \mathrm{CMC} / 0.5 \mathrm{GX} / 0.7 \mathrm{GBA}$ con $22.08 \%$, mientras que los menores valores de humedad (18.21\%) se encontraron en las BP control, es decir aquellas que no contenían GBA. BP con concentraciones de GBA entre 0.1 y $0.5 \%(\mathrm{p} / \mathrm{v})$ tuvieron valores intermedios (20.46 a $21.87 \%$ ). Por lo cual, la GBA podría estar interactuando con la CMC y la GX mediante enlaces de hidrógeno bloqueando la unión de grupos hidroxilos con moléculas de agua, conllevando de esta manera a una disminución del contenido de humedad de las BP (Guo et al., 2014), este comportamiento fue observado en BP con concentraciones de GBA menores a $0.3 \%(p / v)$. Sin embargo, cuando las concentraciones de GBA fueron iguales o superiores a $0.5 \%(\mathrm{p} / \mathrm{v})$ se apreció un incremento en el contenido de humedad, lo cual puede ser consecuencia de una mayor disponibilidad de grupos hidroxilo disponibles para interactuar con moléculas de agua, tal comportamiento también fue reportado por Martins et al., (2012) en BP binarias compuestas por k - carragenato y goma algarrobo. Los aumentos en el contenido de humedad normalmente están asociados al contenido de plastificante utilizado. Por ejemplo, Dick et al., (2015) encontraron incrementos en el contenido de humedad de BP de mucilago de chia de $18.18 \%$ a $41.88 \%$ al incrementar las concentraciones de glicerol. Los grupos hidroxilos junto a las cadenas del plastificante pueden desarrollar enlaces de hidrógeno polímero-plastificante que reemplacen las interacciones polímero - polímero en las BP. Con esto en consideración parece probable que la GBA pueda actuar como plastificante en las BP.

\section{Transparencia de las biopelículas}

La transparencia es una propiedad crítica para las BP comestibles porque afecta la apariencia en general y determina el tipo de aplicación final de la misma. La transparencia de las BP conteniendo $0.7 \%$ (p/v) de GBA (3.32) es mayor que los índices de transparencia reportados por Nur Hazirah et al., (2016) en BP solamente a base de CMC y gelatina (1.99). Estos resultados sugieren que las BP conteniendo GBA son menos transparentes que biopelículas conteniendo CMC y gelatina.

Los valores de transparencia de las BP se muestran en la tabla 1, donde se puede apreciar que un incremento en las concentraciones de GBA de 0.1 a $0.7 \%(p / v)$ aumenta significativamente $(P<0.05)$ los valores de transparencia, indicando que las mayores concentraciones de GBA disminuyen la transparencia de las BP a base de CMC y GX. La mayor transparencia (índice de 1.41) se encontró en las BP sin GBA, seguida por BP con $0.1,0.3,0.5$ y $0.7 \%(p / v)$ con índices de transparencia de 2.67, 2.86, 3.01 y 3.32 respectivamente. Una mayor concentración de GBA disminuye la transparencia de las BP a base de GX y CMC debido al incremento de las reacciones de entrecruzamiento ocasionadas por la presencia de cationes (iones calcio) con los grupos carboxilos disponibles en las hélices de GBA (González et al., 2012). 


\section{Permeabilidad al vapor de agua (PVA)}

La permeabilidad al vapor de agua es un indicador de como la humedad fácilmente atraviesa las BP y se encuentra estrechamente relacionada con reacciones adversas a la vida útil de matrices alimentarias (Ahmadi et al., 2012). Uno de los requerimientos más importantes en las BP comestibles es disminuir la transferencia de humedad entre la matriz alimentaria y su medio ambiente, en tanto, la PVA debe ser lo más baja posible (Kurt y Kahyaoglu, 2014).

En la tabla 1 se observa que los valores de PVA de las BP de CMC y GX incrementan significativamente $(\mathrm{P}<0.05)$ conforme aumenta la adición de GBA $(0.1$ a $0.7 \%$ p/v). Resultados similares fueron obtenidos por González et al., (2016) quieres reportaron incrementos en valores de PVA desde 0.41 hasta 0.88 (g $\mathrm{mm}) /\left(\mathrm{m}^{2} \mathrm{~h} \mathrm{kPa}\right)$ en BP de carragenato cuando aumentaba la concentración de GAA, tal comportamiento es ocasionado por la influencia que ejercen las reacciones de entrecruzamiento sobre el coeficiente de difusión de humedad dentro de la red polimérica indicando cambios estructurales en la matriz polimérica.

La adición de GBA en un $0.7 \%(\mathrm{p} / \mathrm{v})$ exhibe BP con máximos $\left(31.17 \mathrm{~g} \mathrm{~mm} / \mathrm{m}^{2} \mathrm{~d} \mathrm{kPa}\right)$ valores de PVA, contribuyendo de esta manera al incremento en el grado de hidrofilicidad de la BP. Por el contrario, las BP sin la adición de GBA (BP control) presentaron valores de PVA de 20.22 ( $\mathrm{g} \mathrm{mm} / \mathrm{m}^{2} \mathrm{~d} \mathrm{kPa}$ ), mientras que aquellas con concentraciones de GBA entre 0.1 y $0.5 \%(\mathrm{p} / \mathrm{v})$ presentaron valores de PVA entre 21.43 y $28.69\left(\mathrm{~g} \mathrm{~mm} / \mathrm{m}^{2} \mathrm{~d} \mathrm{kPa}\right)$ respectivamente. Estos resultados muestran que la adición de GBA en películas binarias a base de GX y CMC no mejora la PVA; por el contrario, incrementa la permeabilidad al permitir el movimiento de vapor de agua a través de la BP, tal movimiento de vapor es importante en algunos empaques con el fin de evitar los procesos de condensación dentro del empaque. Sin embargo, un aumento en la permeabilidad también incrementa los valores de Aw, lo cual puede propiciar un crecimiento de microorganismos dañinos para la matriz alimentaria (Souza et al., 2010).

Los valores de permeabilidad obtenidos en el presente estudio fueron menores a otras BP poliméricas, incluyendo concentrado de proteína de suero lácteo y mucilago de chia con $0.620-0.678 \mathrm{~g} \mathrm{~mm} / \mathrm{kPa} \mathrm{h} \mathrm{m}^{2}$ (Muñoz et al., 2012), mucilago de nopal con $4.96 \mathrm{~g} \mathrm{~mm} / \mathrm{kPa} \mathrm{h} \mathrm{m}^{2}$, (Espino et al., 2010) y mayores que las

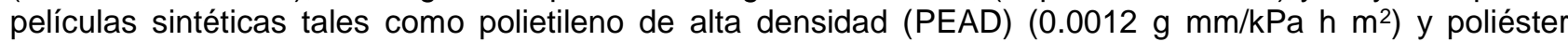
$\left(0.0091 \mathrm{~g} \mathrm{~mm} / \mathrm{kPa} \mathrm{h} \mathrm{m}^{2}\right)$ (McHugh et al., 1993). La disparidad en los resultados entre las BP pueden ser atribuidos a la fuente del hidrocoloide y su proporción en la BP final, el espesor así como diferencias en el proceso de elaboración y análisis. La adición de aceites esenciales se ha visto que aparte de su actividad antimicrobiana pueden reducir los valores de PVA (Ojagh et al., 2010; Pereda et al., 2012).

Tabla 1. Espesor, contenido de humedad, valores de transparencia y PVA de BP conteniendo GBA. Las filas sin ninguna letra en común presentaron diferencias significativas a un nivel de confianza del $95 \%$

\begin{tabular}{|c|c|c|c|c|}
\hline $\begin{array}{c}\text { Proporción de } \\
\text { CMC/GX/GBA (p/v) }\end{array}$ & Espesor $(\mathrm{mm})$ & $\begin{array}{c}\text { Contenido de } \\
\text { humedad }(\%)\end{array}$ & $\begin{array}{c}\text { PVA (g mm/ } \\
\left.\mathrm{m}^{2} \mathrm{~d} \mathrm{kPa}\right)\end{array}$ & $\begin{array}{c}\text { Transparencia a } \\
580 \mathrm{~nm}\end{array}$ \\
\hline $8 / 5 /--$ & $0.16^{\mathrm{a}} \pm 0.01$ & $18.21^{\mathrm{a}} \pm 0.31$ & $20.22^{\mathrm{a}} \pm 1.13$ & $1.41^{\mathrm{a}} \pm 0.18$ \\
\hline $8 / 5 / 0.1$ & $0.17^{\mathrm{ac}} \pm 0.01$ & $21.87^{\mathrm{b}} \pm 0.04$ & $21.43^{\mathrm{a}} \pm 0.88$ & $2.67^{\mathrm{b}} \pm 0.04$ \\
\hline $8 / 5 / 0.3$ & $0.17^{\mathrm{ac}} \pm 0.02$ & $20.46^{\mathrm{b}} \pm 0.12$ & $26.55^{\mathrm{b}} \pm 1.45$ & $2.86^{\mathrm{b}} \pm 0.02$ \\
\hline $8 / 5 / 0.5$ & $0.18^{\mathrm{bc}} \pm 0.01$ & $21.08^{\mathrm{b}} \pm 0.96$ & $28.69^{\mathrm{c}} \pm 0.33$ & $3.01^{\mathrm{c}} \pm 0.26$ \\
\hline $8 / 5 / 0.7$ & $0.18^{\mathrm{bc}} \pm 0.01$ & $22.08^{\mathrm{c}} \pm 0.70$ & $31.17^{\mathrm{d}} \pm 0.71$ & $3.32^{\mathrm{c}} \pm 0.33$ \\
\hline
\end{tabular}

Resistencia a la tensión (RT) y elongación al quiebre (EQ)

Debido que las BP generalmente son sometidas a diferentes esfuerzos externos durante el empacado y acondicionamiento de las matrices alimentarias, sus propiedades mecánicas son un parámetro importante, práctico y tecnológico con el fin de seleccionar los componentes ideales para su elaboración. En este estudio, las propiedades mecánicas fueron determinadas con base a la RT y la EQ. Estos dos parámetros son considerados medidas apropiadas para evaluar la resistencia y flexibilidad de las BP (Kurt y Kahyaoglu, 2014).

En la tabla 2 se presentan los porcentajes de RT y EQ de las BP a base de CMC, GX y GBA. Los valores de RT de las BP control presentaron los menores valores de RT con 9.65 MPa observándose un incremento significativo $(\mathrm{P}<0.05)$ al aumentar las concentraciones de $\mathrm{GBA}$, debido al aumento en las reacciones de entrecruzamiento. Los mayores valores de RT $(25.01 \mathrm{MPa})$ fueron apreciados en BP con $0.7 \%(\mathrm{p} / \mathrm{v})$ seguido por las BP con 0.5 y $0.3 \%$ (p/v) de GBA con 21.00 y $15.49 \mathrm{MPa}$ respectivamente. Resultados similares fueron propuestos por González et al., (2016) en BP a base de GAA y carragenato, donde los valores de RT fueron directamente proporcional a la concentración de GAA. Igualmente, Mostafavi et al., (2016) encontraron valores para RT desde 11.60 a $24.29 \mathrm{MPa}$ en BP a base de goma tragacanto y algarrobo. Estas divergencias son debido a las diferencias estructurales presentes en cada hidrocoloide, lo 
que conlleva a que se puedan establecer una cantidad variable de enlaces de hidrógeno. Ha sido demostrado que las BP naturales o sintéticas con mayor RT usualmente muestran una menor EQ debido a sus características estructurales (Seyedi et al., 2014).

Los valores de RT fueron mayores a los obtenidos por: Dick et al., (2015) en BP de mucilago de chia (9.44 a 17.75 MPa), Espino et al., (2010) en BP a base de nopal (Opuntia ficus-indica L) (0.4-0.95 MPa), Muñoz et al., (2012) para BP conteniendo concentrado de proteína de suero lácteo (2.67 - 4.68 MPa) e incluso fueron superiores a los del polietileno de baja densidad (PEBD) (9-17 MPa) y menores a los del celofan (114 MPa) (Smith, 1986).

Con respecto a los valores de EQ, la adición de GBA $(0.1,0.3,0.5,0.7 \% \mathrm{p} / \mathrm{v})$ disminuye significativamente $(\mathrm{P}<0.05)$ los valores de elongación a las BP binarias desde 79.14 a 73.71, 68.08, 62.23 y 56.71 respectivamente. Este decrecimiento es producido por la estructura de la GBA, la cual forma geles firmes pero quebradizos a entrar en contacto con cationes mono o divalentes. Un comportamiento diferente fue publicado por González et al., (2016) quienes obtuvieron incrementos significativos $(\mathrm{P}<0.05)$ en los valores de $E Q$ cuando incrementaba la concentración de GAA, esta disparidad es producida por la diferencia estructural de las gelanas de alto y bajo acilo (González et al., 2012). Se puede apreciar en términos generales un incremento en la RT y una disminución en EQ. Como resultado del incremento en la concentración de GBA se obtienen BP con menor extensibilidad y mayor resistencia. Resultados contrarios han sido publicados por diversos autores (Ahmadi et al., 2012; Dick et al., 2015) es decir, incrementos en los porcentajes de EQ y disminución en la RT.

Tabla 2. Propiedades mecánicas de las biopelículas binarias conteniendo GBA. Filas sin ninguna letra en común presentaron diferencias significativas a un nivel de confianza del 95\%

\begin{tabular}{|c|c|c|}
\hline Proporción de CMC/GX/GBA (p/v) & RT (MPa) & EQ (\%) \\
\hline $8 / 5 /--$ & $9.65^{\mathrm{a}} \pm 2.73$ & $79.14^{\mathrm{a}} \pm 2.14$ \\
\hline $8 / 5 / 0.1$ & $12.26^{\mathrm{b}} \pm 0.55$ & $73.71^{\mathrm{b}} \pm 1.58$ \\
\hline $8 / 5 / 0.3$ & $15.49^{\mathrm{c}} \pm 1.42$ & $68.08^{\mathrm{c}} \pm 0.43$ \\
\hline $8 / 5 / 0.5$ & $21.00^{\mathrm{d}} \pm 1.35$ & $62.23^{\mathrm{d}} \pm 1.02$ \\
\hline $8 / 5 / 0.7$ & $25.01^{\mathrm{e}} \pm 2.14$ & $56.71^{\mathrm{e}} \pm 1.55$ \\
\hline
\end{tabular}

\section{CONCLUSIONES}

Los resultados obtenidos proporcionan una información interesante sobre algunas propiedades funcionales de las BP a base de CMC, GX y GBA. Las BP con menores concentraciones de goma gelana mostraron propiedades más deseables con respecto a las demás formulaciones, ya que se producen BP con bajo contenido de humedad y PVA, además de presentar elevados valores de EQ y ser más transparentes. Sin embargo, al presentar bajos valores de RT estas BP podrían requerir una cubierta protectora tipo empaque secundario. Estos resultados son realmente prometedores y sugieren la posibilidad de utilizar combinaciones terciarias de CMC, GX y GBA para ser considerado como una fuente de empaque biodegradable para aplicaciones alimentarias.

\section{REFERENCIAS}

Ahmadi, R.; Kalbasi, A. y otros tres autores, Development and characterization of a novel biodegradable edible filmobtained from psyllium seed (Plantago ovata Forsk). J. Food Engineering,109, 745-751 (2012)

American Society for Testing and Materials (ASTM), Standard terminology relating to plastic, Designation D883-00. Annual book of ASTM standards. Philadelphia 13, (1985)

American Society for Testing and Materials (ASTM), Standard Test Methods for Water Vapor Transmission of Materials. West Conshohocken: ASTM, 11, (2010)

Baldwin, E.; R. Hagenmaier y J. Bai, Edible coatings and films to improve food quality, 2nd ed. Boca Raton: CRC Press. (2012)

Bourtoom, T., Edible films and coatings: characteristics and properties, International Food Research Journal, 15(3), 1-12 (2008)

Cao, N.; Y. Fu y J. He, Mechanical properties of gelatin films cross-linked, respectively, by ferulic acid and tannin acid, Food Hydrocolloids, 21, 575-584 (2007) 
Dick, M. y otros cinco autores, Edible film production from chia seed mucilage: Effect of glycerol concentration on its physicochemical and mechanical properties, Carbohydrate Polymers, 130, 198-205 (2015)

Embuscado, M. y K. Huber, Edible film and coating applications. New York: Springer Science Business Media, 430 - 437 (2009)

Espino, M. y otros siete autores, Development and characterization of edible films based on mucilage of Opuntia ficus-indica (L.), Journal of Food Science, 75(6), E347-E352 (2010)

Ghanbarzadeh, B. y H. Almasi, Physical properties of edible emulsified films based on carboxymethyl cellulose and oleic acid, International Journal of Biological Macromolecules, 48, 44-49 (2011)

Ghasemlou, M.; Khodaiyan, F. y otros dos autores, Development and characterization of new biodegradable edible film made from kefiran, an exopolysaccharide obtained from kefir grains, Food Chemistry, 127, 1496$1502(2011)$

González, R. y otros tres autores, Rheological characterization and activation energy values of binary mixtures of gellan, European Food Research Technology, 235, 305-313 (2012)

González, R.; L. Morón y A. Tarón, Caracterización físico mecánica de biopelículas comestibles binarias a base de $k$ - carragenato y gelana de alto acilo, Vitae 23 (Supl. 1), S185 - S189 (2016)

Guo, J. y otros cuatro autores, Periodate oxidation of xanthan gum and its crosslinking effects on gelatinbased edible films, Food Hydrocolloids, 39, 243-250 (2014)

Hager, A.; Vallons, K.; K. Arendt, Influence of gallic acid and tannic acid on the mechanical and barrier properties of wheat gluten films, Journal of Agricultural and Food Chemistry, 60, 6157-6163 (2012)

Khazaei, N.; Esmaiili M. y otros tres autores, Characterization of new biodegradable edible film made from basil seed (Ocimumbasilicum L.) gum, Carbohydrate Polymers, 102, 199-206 (2014)

Kokoszka, S.; Debeaufort, F. y otros tres autores, Protein and glycerol contents affect physico-chemical properties of soy protein isolate-based edible films, Innovative Food Science \& Emerging Technologies, 11, 503-510 (2010)

Kurt, A. y T. Kahyaoglu, Characterization of a new biodegradable edible film made from salep glucomannan, Carbohydrate Polymers, 104(0): 50-58 (2014)

Martins, J. y otros cinco autores, Synergistic effects between k-carrageenan and locust bean gum on physicochemical properties of edible films made thereof, Food Hydrocolloids, 29, 280-289 (2012)

McHugh, T.; Avena, R. y otros dos autores, Hydrophilic edible films:Modified procedure for water vapor permeability and explanation of thicknesseffects, Journal of Food Science, 58(4), 899-903 (1993)

Mostafavi, S. y otros tres autores, Preparation and characterization of tragacanth-locust bean gum edible blend films, Carbohydrate Polymers, 139 (2), 20-27 (2016)

Muñoz, L.; Aguilera, J. y otros dos autores, Characterization and microstructure of films made from mucilage of Salvia hispanicaand whey protein concéntrate, Journal of Food Engineering, 111(3), 511-518 (2012)

Nur Hazirah, M.; N. Isab y M. Sarbona, Effect of xanthan gum on the physical and mechanical properties of gelatin-carboxymethyl cellulose film blends, Food Packaging and Shelf Life, 9, 55-63 (2016)

Ojagh, S.; Rezaei, M. y otros dos autores, Development and evaluation of a novel biodegradable film made from chitosan and cinnamon essential oil with low affinity toward water, Food Chemistry, 122(1), 161-166 (2010)

Pérez, P.; Reina, J. y otros tres autores, Packaging properties and control of Listeria monocytogenes in bologna by cellulosic films incorporated with pediocin, Brazilian Journal of Food Technology, 16(3), 226-235 (2013)

Pereda, M.; G. Amica y N. Marcovich, Development and characterization of edible chitosan/olive oil emulsion films, Carbohydrate Polymers, 87(2), 1318-1325 (2012) 
Seyedi, S.; A. Koocheki y otros dos autores, Lepidium perfoliatum seed gum: A new source of carbohydrate to make a biodegradable film, Carb Polym, 101(0): 349-358 (2014)

Smith, S., Polyethylene, low density. In M. Bakker (Ed.), The Wiley encyclopedia of packaging technology New York, NY: John Wiley and Sons, 514-523 (1986)

Souza, B.; M. Cerqueira y otros dos autores, The use of electric fields for edible coatings and films development and production: a review, Food Engineering Reviews, 2, 244-255 (2010)

Su, J.; Z. Huang y otros tres autores, Structure and properties of carboxymethyl cellulose/soy protein isolate blend edible films crosslinked by Maillard reactions, Carbohydrate Polymers, 79, 145-153 (2010)

Yang, L.; A. Paulson, y M. Nickerson, Mechanical and physical properties of calcium-treated gellan films, Food Research International, 43, 1439-1443 (2010) 